\section{Variety at Nature conference}

Plants engineered to produce antibodies against viral pathogens, protein frameworks into which various of functional units can be plugged at will and biosensors based on a marriage of immunology and fibre optics were among the more engaging ideas to emerge from Nature's "New Technology in Biotechnology" conference at London's Novotel last week.

Clearly the path to commercial reality from an engaging biotechnological idea is long and exceedingly stony, but it is increasingly travelled. Ten years after the discovery of hybridoma technology, John Birch could talk of the production at Celltech of around a kilogram of monoclonal antibody a year, mostly for blood group testing, (see Nature 27 June, p.705) and its sale for, typically, $£ 1,000$ a gram.

Clever variations on the basic theme of monoclonal antibodies are already proving their worth at the Molecular Research Council's Laboratory of Medical Biology in Cambridge. Most are the result of engineering the genes for antibodies, replacing an inessential part of the sequence with another gene. The results are hybrid, bifunctional proteins, several of which were described by Michael Neuberger. In one, the substitute gene is for an enzyme used in DNA sequencing. After the hybrid antibody/enzyme is produced in large quantities by standard hybridoma technology, it is very simply and efficiently immunopurified by means of its antibody end; the other end is now supplying the laboratory's considerable demand for the enzyme. On the other side of the coin, a protein that is unable to induce good monoclonal antibodies has been engineered to contain an extra short sequence so that it can be recognized by an existing and excellent monoclonal antibody against the short sequence.

Daniel Thomas of the University of Compiègne foresaw the value of hybrid enzymes in second-generation immobilized enzyme technology. For example, the lifetime of an oxidase that is susceptible to damage by some of the oxygen radicals it produces could be lengthened if the ox-

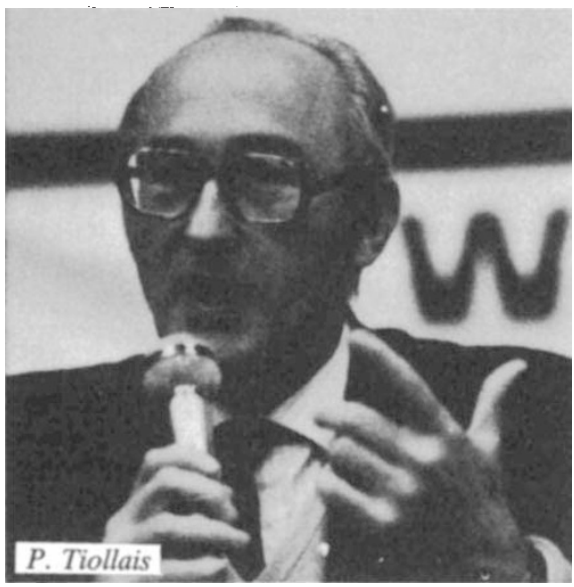

idase were to be hybridized with a superoxide dismutase, which can consume the radicals. Other important second generation systems would, for example, have built-in systems of co-factor regeneration on electrochemical surfaces or would operate in organic solvents, perhaps to reverse an enzyme reaction or in organic synthesis. More sophisticated protein engineering will involve the subtle redesign of active sites of enzymes, which will remain very much a matter of trial and error until much more is known of the determinants of protein folding, according to Greg Winter of the Medical Research Council Laboratory of Molecular Biology in Cambridge.

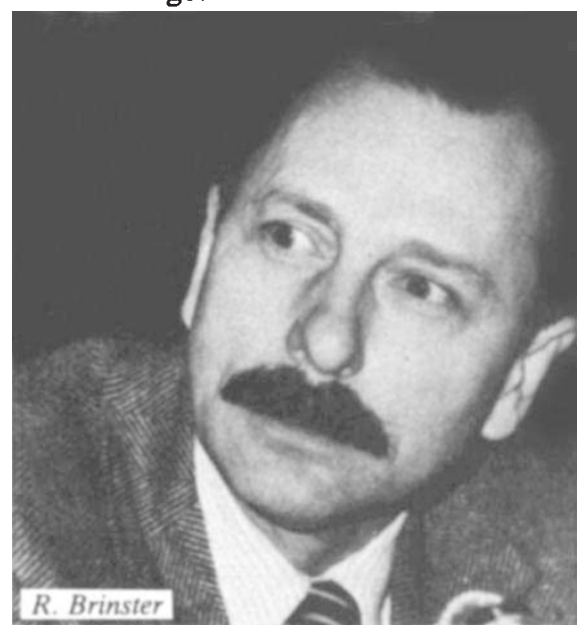

Even without engineering, enzymes and antibodies have many projected uses in biosensors, very few of which have yet progressed from the laboratory to the market place. Michael Flanagan of University College London described prototype optical immunosensors in which the binding of fluorophore-labelled antigen to antibodies immobilized on a surface of the device is measured by means of evanescent light waves of plasmon resonance laser light. For potentiometric devices, enzyme immobilization techniques badly need adapting to suit the surface of microelectronic devices, he said. For amperometric devices, John Higgins of Cranfield Institute of Technology and Leicester Biocentre described current interest both in the organometallic ferrocenes as mediators of electron transfer to electrodes from enzymes bound to them and in a newly discovered class of dehydrogenase enzymes that use pyrroloquinolinequinone as a cofactor in place of the more usual but problematic nicotinamide adenine dinucleotide.

Recombinant DNA technology lags behind that of hydridomas and perhaps even biosensors in its commercialization. But there are products in the offing. Pierre Tiollais of the Pasteur Institute described the advanced state of a hepatitis $B$ vaccine from engineered mammalian cells and Art

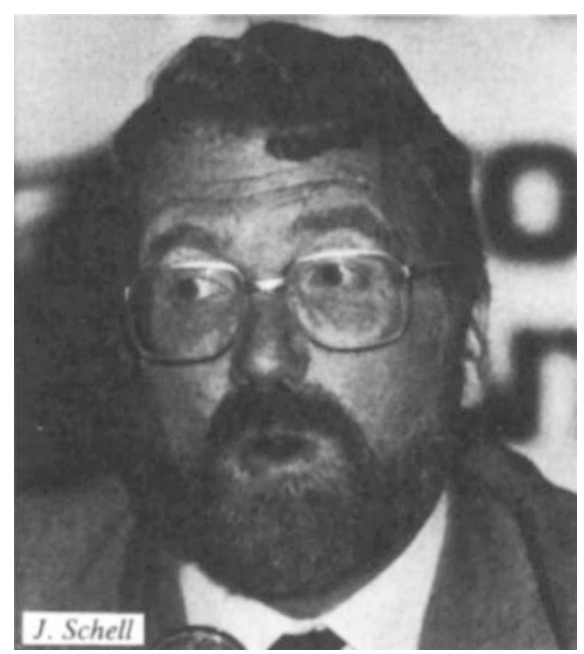

Levinson of Genentech claimed that the company's tissue plasminogen activator has been able to dissolve blood clots and restore blood flow through the coronary arteries of some patients in a clinical trial.

For plant breeders, the most immediate gain is likely to be strains that have been engineered to be herbicide resistant, according to Jozeph Schell of Ghent University and the Max Planck Institute for Plant Breeding Research in Köln. Less advanced are attempts to improve potato protein quality and quantity, to transfer light-inducibility to new genes and to engineer cereal crops. And but a glint in Schell's eye is a plan to give tobacco plants the mammalian gene necessary to produce a protective antibody against tobacco mosaic virus. For animal breeders, Ralph Brinster of the University of Pennsylvania offered encouraging news of the general applicability of the introduction of genes into fertilized eggs and the tissue-specific expression of the genes at high levels in the subsequent transgenic animals. One recently learnt fact is that the removal of residual bacterial sequences from a gene construction introduced into the eggs can greatly increase the gene's expression in the tissues of the transgenic animals.

Peter Newmark

For those who would like a record of the Nature conference, cassette tapes are available from: Audio Bass Ltd, 16 Longland Drive, Totteridge, London N20 8HE, UK (Tel: 01-445 6776 or 01-349 4041). Please quote programme number 064-85.

The correct answers in the exhibition competition were as follows:

1. Tissue culture bottle/Laminar flow hood

2. Eppendorf microfuge

3. Dilson pipetman

4. Scintillation counter

5. Tube sealer

6. Fibre optics

None of the entrants identified all the photographs correctly. Sally Roberts and Pam Jones, both of the Laboratory of Molecular Biophysics at Oxford, got five out of the six right, and in the draw Sally Roberts was selected as the winner of the personal computer. 